\title{
Cystic dilatation of ventriculus terminalis: report of two cases
}

\begin{abstract}
Ventriculus terminalis is a space which is coated by ependymal cells in conus medullaris of medulla spinalis. It is formed during the embryonic development, also known as the ependymal cyst or the 5th ventricle. This rare entity in adults can be detected on thoracolumbar MR images. It is useful to know the intensity of the image in distinguishing it from the cystic tumors located in the same region. Two middle-aged female patients referred with low back pain complaint and thoracolumbar region images obtained by routine magnetic resonance imaging sequences. The MR images revealed the cystic dilatation of ventriculus terminalis with a well-defined outline, with no septation, cord edema and additional spinal abnormality.
\end{abstract}

Volume 7 Issue 3 - 2018

\author{
Adem Yokuș, Sercan Özkaçmaz, Suat Orak \\ Van Training and Research Hospital, Clinic of Radiology, Turkey
}

Correspondence: Adem Yokuș, Van Training and Research Hospital, Clinic of Radiology, 65080,Van, Turkey, Tel 0 (432) 215 760I/0536 34532 56, Fax: 0 (432) 2121954 ,

E-mail drademyokus@gmail.com

Received: April 12, 2018 | Published: May 08, 2018

Keywords: ventriculus terminalis, cystic dilatation, magnetic resonance imaging

\section{Introduction}

Ventriculus terminalis (VT) or fifth ventricle is a space filled with cerebrospinal fluid (CSF) which is coated by ependymal cells in conus medullaris of medulla spinalis. It was first described by Stilling in 1859. In 1875 Krause used the term "fifth ventricle" after observing that the walls of the cavity consisted of normal ependymal cells. It has been reported in the literature that the dilated VT is seen in $2.6 \%$ of pediatric patients under five age, but observed rarely in adults. ${ }^{1-3}$ The etiology and pathogenesis of isolated dilatation of VT is still uncertain. Various hypotheses have been proposed to explain the pathophysiology of VT. It is proposed that cavitations may be associated with trauma, vascular disorders, inflammatory or compressive pathologies of the spinal cord. ${ }^{4,5}$ An important part of the cystic lesions seen in the conus medullaris are tumors. Therefore, it is important to recognize the radiological features of VT. ${ }^{5}$ In this presentation, we report magnetic resonance imaging (MRI) findings of cystic dilatation of VT in two middle-aged female patients with low back pain complaint.

\section{Methods}

Magnetic resonance imaging was performed using 1.5T MR system with a surface coil. Sagittal and axial images were obtained with spin-echo T1-weighted sequences and T2-weighted sequences. The first patient received contrast agent.

\section{Case I}

MRI was performed in a 33-year-old female patient with a low back pain complaint. Thoracolumbar region images were obtained. On sagittal and axial T2-weighted images, a cystic dilatation with dimensions of $11 \times 8 \mathrm{~mm}$ which is isointense with CSF in all MRI sequences was detected in the centre of medulla spinalis at conus medullaris toracic 12 - lumbar 1 vertebrae level (Figure 1A), (Figure 1B). Pre-contrast and post-contrast T1-weighted images showed no contrast enhancement in the lesion (Figure 2A), (Figure 2B). The signal intensity of medulla spinalis was observed to be normal, perilesional cord edema was not detected. There was no septation within the detected lesion. There were no additional spinal anomalies such as hydromyelia cavity and vertebral deformities that could accompany the lesion.
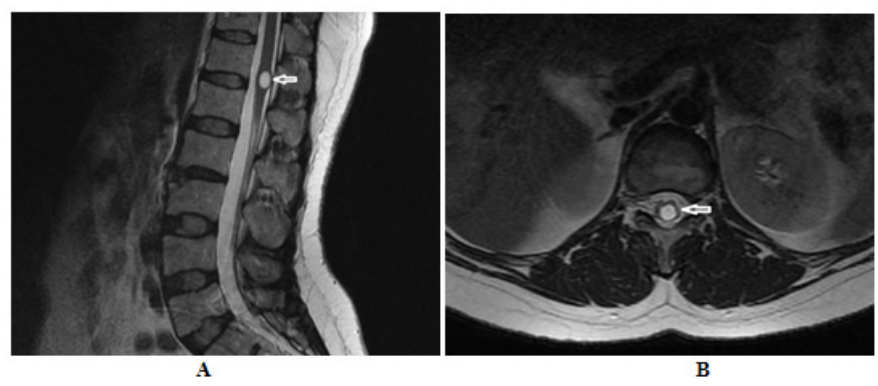

Figures 1 (A) \& 1(B) Sagittal and axial T2 weighted images show cystic dilatation that is isointense with CSF in conus medullaris at the level of T12-L1 vertebrae.
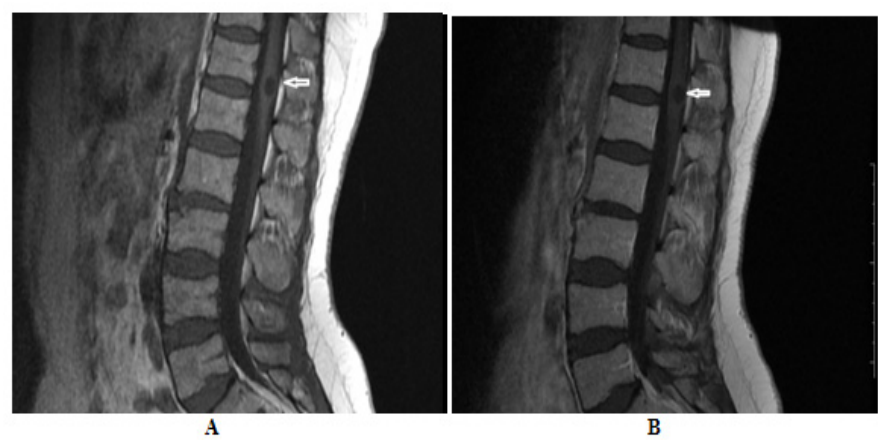

Figures 2(A) \& (B) Sagittal T1 weighted images; contrast enhancement was not detected on pre-contrast (A) and post-contrast (B) T1-weighted images.

\section{Case 2}

Thoracolumbar region images obtained from routine MR sequences of a 30-year-old female patient with a complaint of low back pain were examined. On sagittal T1 and T2-weighted and axial T2-weighted images, a cystic dilatation with dimensions of $9 \times 4,5 \mathrm{~mm}$ which is isointense with CSF in all MRI sequences was detected in the centre of medulla spinalis at conus medullaris lumbar 
1 vertebrae level (Figure 3A), (Figure 3B). The signal intensity of medulla spinalis was observed to be normal, perilesional cord edema was not detected. There was no septation within the lesion. There were no additional spinal anomalies such as hydromyelia cavity and vertebral deformities that could accompany the lesion. No contrast enhancement examination was performed because the patient had contrast allergy.

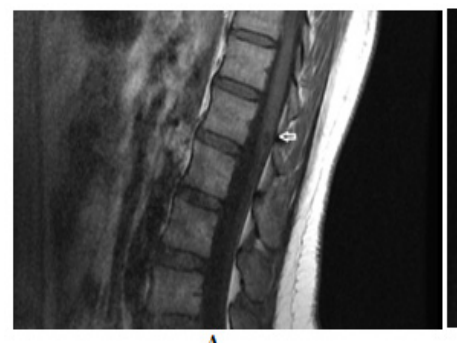

A

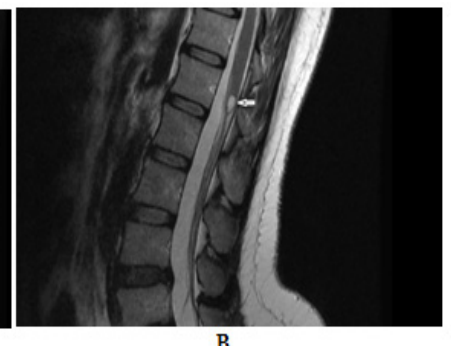

B
Figures 3(A) \& (B) Sagittal T1 and T2-weighted and axial T2-weighted images, a cystic dilatation which is isointense with CSF in all MRI sequences in the centre of medulla spinalis at conus medullaris (L1 vertebrae) level.

\section{Discussion}

Ventriculus terminalis is a cavity covered with ependymal cells in conus medullaris. The normal development of fetal medulla spinalis occurs in two different stages. During the neuralation stage, the medulla spinalis is shaped by the closing of the neural tube. The extreme caudal portion of the medulla spinalis, also called conus medullaris, develops secondary, this different stage is called canalization with retrogressive differentiation. At this stage, the conus medullaris, filum terminale and VT all develop from the caudal cell mass which consist of undifferentiated cells. The VT has been defined as a normal developmental phenomenon in newborns and pediatric cases; but it is a rare pathology in adults and few cases have been reported in the literature. ${ }^{4,6,7}$ Coleman et al. ${ }^{2}$ reported that there is no pathology associated with VT and they reported also VT has no pathological prognosis in their study on 418 children's MR images. In the same study, the incidence of childhood VT was found to be $2.6 \%$ under the age of five. Suh et al. ${ }^{4}$ reported that septation, perilesional cord edema, kyphosis and arteriovenous malformation were associated with ventriculus terminalis in a study of 10 adult patients with VT. In our cases, no significant additional pathology was detected.

In adults, VT is rarely encountered in thoracolomber MR images. Cystic neoplasms located at the same level take place in the differential diagnosis, therefore the image feature and intensity of the lesion must be known. The most common tumors in the conus medullaris are ependymomas, astrocytomas and oligodendrogliomas. Intramedullary neuromas and hemangioblastomas are rare. In cystic tumors of the conus medullaris, it is expected that the solid portion of the tumors show a contrast enhancement. On the contrary, the cystic dilatation of the VT does not enhance after contrast injection. ${ }^{5}$ The cystic dilatation of the VT is seen as an ovoid-shaped, regular wall structure in MR images, with no septation and continuing with canalis centralis. The intensity of fluid within the lesion is hypointense on T1-weighted images, hyperintense on T2-weighted images, and isointense with CSF in all MRI sequences. No contrast enhancement is found within the cyst, on the wall or in the surrounding tissues. ${ }^{1,5,8} \mathrm{VT}$ cases are usually asymptomatic and the treatment is based on clinical findings. Patients with nonspecific symptoms are followed up by conservative treatment; however, surgical treatment may be required in cases with focal neurological deficits. ${ }^{4}$ Furthermore Suh et al. ${ }^{4}$ remarked that, it is likely that the degree of clinical symptoms are related to the size of the conus lesion. Therefore, we think that asymptomatic cases and patients with nonspecific complaints should be followed up with MRI.

\section{Conclusion}

MR imaging can diagnose cystic dilatation of the VT and can exclude a tumor. It can also detect pathologies that may accompany the lesion. Therapeutic management can be determined according to clinical symptoms and MR imaging assessment.

\section{Acknowledgment}

None.

\section{Conflict of interset}

Authors declare that their is no conflict of interest.

\section{References}

1. Fazlığulları Z, Uysal İI, Kıvrak AS, et al. Ventriculus terminalis' in kistik dilatasyon olgusunda MR görüntüleme bulguları. Med J SDU/SDÜ Tip Fak Derg. 2015;22(2):45-48.

2. Coleman LT, Zimmerman RA, Rorke LB. Ventriculus terminalis of the conus medullaris: MR findings in children. AJNR Am J Neuroradiol. 1995;16(7):1421-1426.

3. Liccardo G, Ruggeri F, De Cerchio L, et al. Fifth ventricle: an unusual cystic lesion of the conus medullaris. Spinal Cord. 2005;43(6):381-4.

4. Suh SH, Chung T, Lee S, et al. Ventriculus Terminalis in Adults: Unusual Magnetic Resonance Imaging Features and Review of the Literature. Korean J Radiol. 2012;13(5):557-63.

5. Sigal R, Denys A, Halimi P, et al. Ventriculus terminalis of the conus medullaris: MR imaging in four patients with congenital dilatation. $A J N R$ Am J Neuroradiol. 1991;12(4):733-7.

6. Kriss VM, Kriss TC, Babcock DS. The ventriculus terminalis of the spinal cord in the neonate: a normal variant on sonograph. AJR Am J Roentgenol. 1995;165(6):1491-3.

7. Kriss VM, Kriss TC, Coleman RC. Sonographic appearance of the ventriculus terminalis cyst in the neonatal spinal cord. J Ultrasound Med. 2000;19(3):207-9.

8. Ganau M, Talacchi A, Cecchi PC, et al. Cystic dilation of the ventriculus terminalis. J Neurosurg Spine. 2012;17(1):86-92. 